\title{
Risk-Aware Scheduling For Multi User Video Streaming Through Omap 3530 Using Linux
}

\author{
K.M.Padmini ${ }^{1}$, Mrs.R.Geetha ${ }^{2}$ \\ ${ }^{1} E E E$, Ganadhipathy tulsi's Jain engineering college, Anna university, India \\ ${ }^{2}$ EEE, Ganadhipathy tulsi's Jain engineering college, Anna university, India
}

\begin{abstract}
Now a days live video streaming becomes more popular in the residential market. As the number of users increases the requirement of bandwidth also increases. The quality of video is affected due to the limitation of bandwidth. So far, protocol RTSP has been used for the transmission that supports retrieval of media from server and also supports recording of conference. In this paper, we propose an Enhanced Media Codec protocol for the transmission of high quality video without pixel drops according to the resolution size of the customer's monitor.
\end{abstract}

Keywords: Enhanced Media codec protocol, Designated Rendezvous Point, beagle board revision c3, Linux; Video streaming, Roadside access Point.

\section{Introduction}

HTTP Live Streaming (HLS) protocol supports live and on demand streaming using an ordinary web server. It has the ability to adapt the quality to device and network condition automatically.

Live stream is segmented and stored on web server before delivery. During playback, the player reassemble the segments seamlessly.If multiple qualities of streams are available, the players monitor the available bandwidth continuously and pick the next fragment from the highest quality. This is called adaptive streaming. Most popular web player is JW player that supports HTTP live streaming.

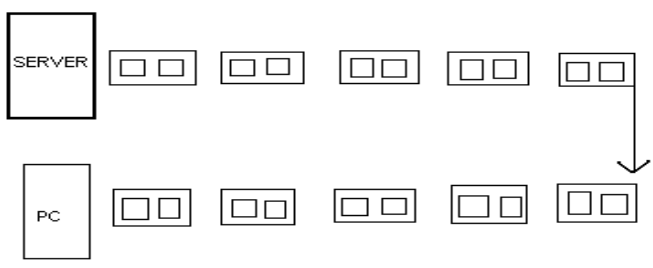

Fig 1. Video streaming

[1] Research has made based on Delay constrained rate adaptation algorithm and is used to select an optimal bit rate at IEEE802.11b. Simulation output based on NS-2 shows that there is an possibilities of better quality rate adaptation. [2]Research made on Link adaptation and cross layer signaling and is used for the quality of video. During transmission of videos, SNR related information is used to change the link conditions. Statistics based automatic rate control is used to maintain frame error rate, ACK transmission and achieve throughput. Hybrid automatic rate control is used to maximize throughput but its slow response to changes of link condition. Cross layer signaling is ineffective when round trip delays are too long. video encoder is used at cross layer signaling.H.263 encoder supports video rate control algorithm to achieve quantization step size. [3]In streaming media system, a server pre-stores encoded media data and transmit it on demand to a client for playback in real time. Client buffers the data that it receives and begins playback after a short delay of upto several seconds. This delay is fixed and does not depend on the length of presentation. Window control can be used to spread out transmission over the duration of the session. Different data units are given different windows of transmission based on their delivery deadlines. [4] Distributed algorithm for channel time allocation is used among multiple video streams for the transmission. Heuristic packet pruning scheme is used to achieve HD quality. To achieve higher data rate H.264/AVC is used for video encoding. Data rate is achieved up to 6-12 Mbps during transmission. [5] Later research had made on Multiple description coders and are used in both balanced and unbalanced operation. Thus, Bandwidth in each path may be similar or different resulting in balanced or unbalanced operation. Internet is hampered by limited Bandwidth and packet loss. So MD encoder is used to overcome this problem.[6]In streaming technology, Multiple description coding is used in which odd/even frames sent on separate path. After transmitting the frames in different path Reference picture selection scheme is used in which damaged frames are predicted to retransmit the lost packet over base layer. RTP is used for data transfer. [7]Research has made based on Multiple tree construction protocol and is widely used which builds two nearly disjoint tree. Here, Quality of video is superior than single tree multicast. Multiplr tree construction protocol is widely used in VLAN and packets are passed between one or two routers and transmission is over 
multicast. Multiple tree protocol constructed in parallel and in distributed fashion in order to reduce time and control overhead it takes to construct trees.Before transmission the Tree flag is set at packet header to determine which tree packet should be forwarded. In Single tree multicast protocol, it uses JQ(join query) and JR(join reply) messages to sender for constructing multiple tree. [8] In streaming technology Single tree protocol is used for transmission of high quality video. Routing algorithm is used to identify the shortest path to reach the destination.NS-2 simulator is used for the simulation and the output shows the high quality videos. Congestion optimized traffic partitioning scheme is used to avoid traffic during transmission. In that Video distortion model helps to incorporate contributions from both encoder and packet loss due to congestion.

\section{Block Diagram}

The input video file is given at input block for the transmission. JW player automatically selects the best playback for viewer's device like smart phone, desktop, or tablet. It supports live and on demand adaptive streaming. The protocol provides a set of rules for transmitting the given input video files. Web link is used for transmitting videos over internet and its multicast to various users.

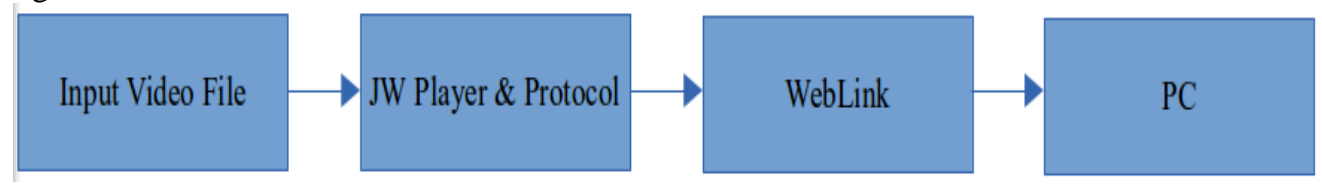

Fig 2. Block diagram

\section{Enhanced Media Codec Protocol}

The EMC protocol is used for the video transmission that transmits the video frames from server to various client through designated rendezvous point (DRP) and feedback rooter (FBR).This protocol assigns the client to particular tree based on the network conditions and availability of bandwidth to achieve the higher throughput .

The bit rate is selected based upon the following criteria,

Key frame interval of your encodings,
Buffer length configuration of your player, and
Dit rate spacing and quantity of your media files
Feedignated Rendezvous Point (DRP)
Forward Branch
Feedback Root

The procedure of enhanced media codec protocol is as follows

Step 1: The client which request for video streaming to be played on the browser request the video streaming server through http request message.

Step 2: Once the server receives request from client, server request for aunthenticity conformation.

Step 3: Once client gets authenticated, server sends the list of encoding bit rate. This has been updated by client through status code 100 about the network conditions on http client status code 1xx series.

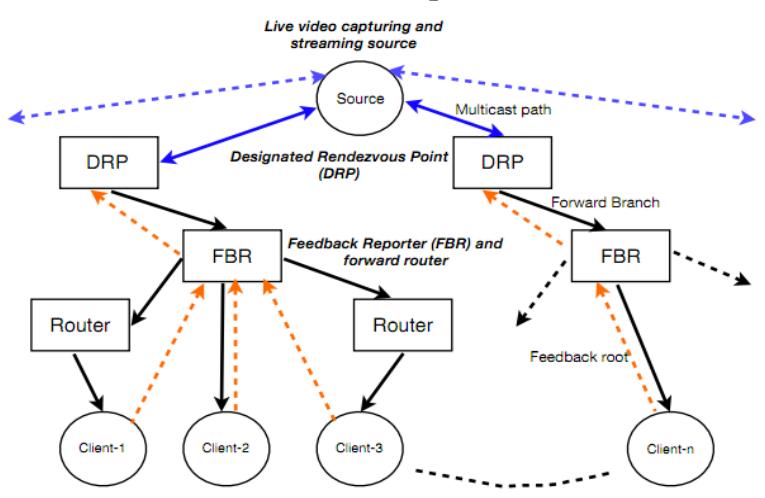

Fig 3.Transmission procedure

Step 4: After receiving status code from client, server open the Feedback reporter and assigns client to specific group of tree based on data rate and bandwidth availability.

Step 5: The assigned DRP and FBR socket details will be send through status code 200.

Step 6: After client receiving message code, opes two separate socket one for DRP incoming message and FBR outgoing status code. 
Step 7: Client once again request for video packets to DRP through FBR outgoing status code.

Step 8: Server once received request from DRP it initiate live streaming content to client through DRP.

\section{Step 1: Dynamic node selection}

\section{Video Distribution}

In this step, packets are generated at MAC layer and the gateway is responsible for the identification of relay nodes. Each newly selected relay node repeats the procedure till all the neighbor relay nodes scheduled. This procedure is described as children selection procedure. The parent and the children node selection are based on the mobility. And it is controlled by RAP[Roadside Access Point].

The children node that are scheduled by relay node changes with time. Thus it is dynamic process. The relay node will partition the surrounding space in four identical sector at 90 degree in which center of them is parent node, remaining three is children sector. If the children sector is not scheduled, the relay node forwards the slot content that contains video frame sequence number which is smaller or equal to the already received frames. If more than two children are selected, the relay will transmit based on the "grid coloring" scheme.

\section{Step 2: Segmentation of video}

In this step, music or video clips are transmitted on the data channel and it is in fixed length time frame. The time frame is divided into equal slots. Each node includes segmentation and reassembly layer so that each frame is segmented and transmit it as several video frames with different size as I,B or P frames.

\section{Step 3: Streaming of video}

The video that is transmitted is in sequence within its allocated slot and it appends the information at MAC layer header as follows

* Number of bits.

- Identity of three children.

* Slot number of the child that is scheduled.

There is no explicit ACK from the children node. Only a passive ACK is obtained at the relay nodes. If the children transmission is correct the traffic relaying is successful.

\section{Hardware Description}

The Beagle Board is an OMAP3530 platform designed specifically to address the Open Source Community. the OMAP3530DCBB72 720MHZ version and comes in a .4mm pitch POP package. POP (Package on Package) is a technique where the memory, NAND and SDRAM, are mounted on top of the OMAP3530.The Micron POP memory is used on the Rev C4 Beagle-board and is mounted on top of the processor. The key function of the POP memory is to provide 2Gb NAND x 16 (256MB) and 2Gb MDDR SDRAM x32 (256MB @ 166MHz) No other memory devices are on the Beagle Board. It is possible however, that additional memory can be added to Beagle Board by Installing a SD or MMC in the SD/MMC slot, Use the USB OTG port and a powered USB hub to drive a USB Thumb drive or hard drive. The TPS65950 is used on the Rev C4 to provide power to the Beagle Board with the exception of the $3.3 \mathrm{~V}$ regulator which is used to provide power to the DVI-D encoder and RS232 driver.

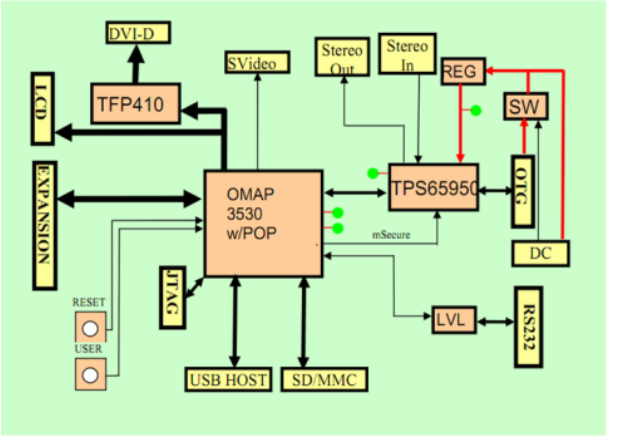

Fig.4.Beagle Board

The USB OTG port can be used as the primary power source and communication link for the Beagle Board and derives power from the PC over the USB cable. The client port is limited in most cases to $500 \mathrm{~mA}$ by the PC. A single PC USB port is sufficient to power the Beagle Board. If additional devices are connected to the expansion bus and the $5 \mathrm{~V}$ rail is used to power them or if a high powered USB device is connected to the EHCI port, then the power required could exceed that supplied by a USB port or Hub.

A 4 pin DIN connector is provided to access the S-Video output of the Beagle Board. This is a separate output from the OMAP processor and can contain different video output data from what is found on the DVI-D 
output if the software is configured to do it. It will support NTSC or PAL format output to a standard TV. The default is NTSC, but can be changed via the Software.

\section{Software Description}

System software based on embedded Linux. The device has a control system of real time collection, whose requirement of the real time of the system is very high therefore we need to adapt the Linux operating system which is based on the high real-time as the software of the device.

FreeRTOS is a popular real time operating system for embedded devices. The kernel itself consists of oly three or four $\mathrm{C}$ files. To make code readable, easy to port and maintainable it is written mostly in $\mathrm{C}$ but there are few assembly functions included where needed. FreeRTOS provides methods for multiple threads or tasks, mutexes, semaphores and software timers.

\section{Beagle Board Boot Process:}

* Before intiating the boot process there is a need of the XLoader and U-Boot source.

* FreeRTOS sources and headers (croutine.c, list.c, queue.c, tasks.c, include folder)

* FreeRTOS ports for ARM - (port.c, portASM.S startup.S, portmacro.h, portmacro.inc, FreeRTOSConfig.h)

* FreeRTOS memory manager - (heap_2.c)

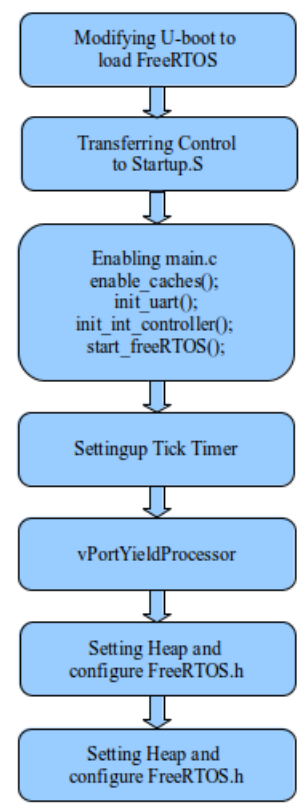

Fig.5.Porting Process

\section{Simulation}

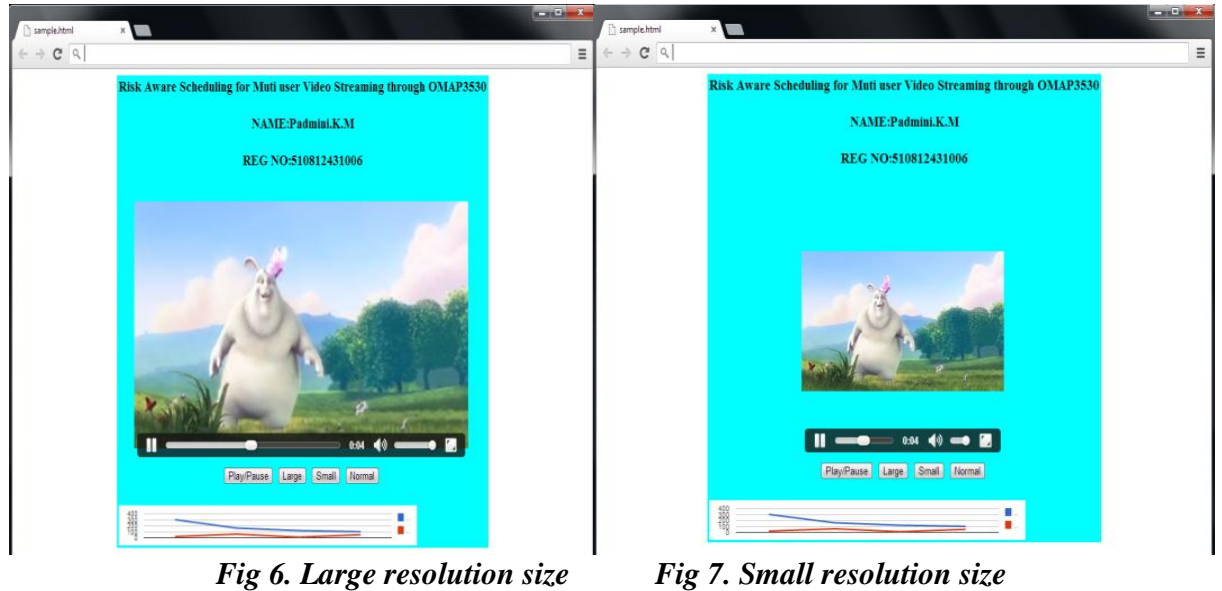




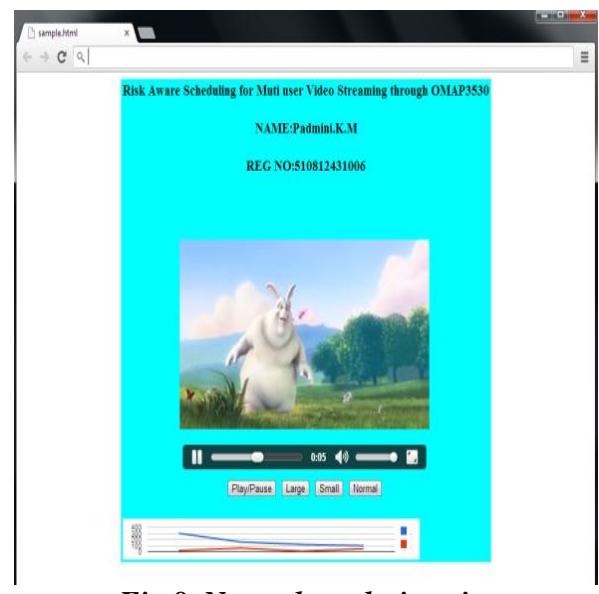

Fig 8. Normal resolution size

The results showed the quality of video according to the resolution size of the viewer's monitor. The first result shows video transmission for large resolution size. The second results shows resolution for small size. Finally, third result shows resolution for normal resolution size of monitor.

\section{Conclusion}

The issue of on-board live video streaming is addressed and a solution is provided, using our protocol, enhanced media codec. The content distribution is achieved through a fully distributed, dynamic selection of forwarders, which acts as a local broadcaster. Graph coloring is used to solve the scheduling problem and optimize the special separation of concurrent streaming transmissions.

\section{References}

[1]. P.van Beek and M.U.Demircin, "Delay-constrained rate adaptation for robust video transmission over home net-works", IEEE International Conference on Image Processing, (ICIP’05), Genova, Italy, vol.2, pp.173 176, Sept.2005.

[2]. L.Haratcherev, J.Taal, K.Langendoen, R.Lagendijk, and H. Sips, "Optimized video streaming over 802.11 by crosslayer signaling", IEEE Communications Magazine, vol. 44,no.1, pp. 115- 121, Jan. 2006

[3]. M.Kalman and B.Girod, "Optimal channel-time allocation for the transmission of multiple video streams over a shared channel", Proc.IEEE International Workshop on Multimedia Signal Processing (MMSP’05), Shanghai, China, pp. 1-4,Oct. 2005

[4]. X.Zhu, P.van Beek, and B. Girod, "Distributed channel time allocation and rate adaptation for multi-user video streaming over wireless home networks”, IEEE International Confer-ence on Image Processing (ICIP’07), Accepted, 2007

[5]. J.G.Apostolopoulos and S.J.Wee, "Unbalanced Mul-tiple Description Video Communication Using Path Diver-sity", IEEE International Conference on Image Processing (ICIP’01), Thessaloniki, Greece, vol.1, pp. 966-969, Oct.2001

[6]. S.Mao, S.Lin, S.Panwar, Y.Wang, and E.Celebi, "Video transport over ad hoc networks:Multistream coding with mul-tipath transport", IEEE Journal on Selected Areas in Commu-nications, vol. 21, no. 10, pp. 1721-1737, Dec. 2003.

[7]. W.Wei and A. Zakhor, "Multiple tree video multicast over wireless ad hoc networks", IEEE Trans.on Circuits, Systems and Video Technology, vol.17, no.1, pp.2-15,Jan. 2007

[8]. E.Setton, X.Zhu,and B.Girod, "Congestion-optimized multipath streaming of video over ad hoc wireless networks", Proc.IEEE International Conference on Multimedia and Expo (ICME’04), T aipei, Taiwan, vol.3, pp.1619-1622, July 2004. 